CCNY-HEP-14/1

March 2014

\title{
Exact operator Hamiltonians and interactions in the droplet bosonization method
}

\author{
Dimitra Karabali ${ }^{a}$ and Alexios P. Polychronakos ${ }^{b}$ \\ ${ }^{a}$ Department of Physics and Astronomy \\ Lehman College of the CUNY \\ Bronx, NY 10468 \\ ${ }^{b}$ Physics Department \\ City College of the CUNY \\ New York, NY 10031 \\ E-mail: dimitra.karabali@lehman.cuny.edu \\ alexios@sci.ccny. cuny. edu
}

\begin{abstract}
We derive the exact form of the bosonized Hamiltonian for a many-body fermion system in one spatial dimension with arbitrary dispersion relations, using the droplet bosonization method. For a single-particle Hamiltonian polynomial in the momentum, the bosonized Hamiltonian is a polynomial of one degree higher in the bosonic 'boundary' field and includes subleading lower-order and derivative terms. This generalizes the known results for massless relativistic and nonrelativistic fermions (quadratic and cubic bosonic Hamiltonians, respectively). We also consider two-body interactions and demonstrate that they lead to interesting collective behavior and phase transitions in the Fermi sea.
\end{abstract}




\section{Introduction}

Bosonization, the description of fermion systems in terms of bosonic degrees of freedom, is a well-established and useful technique, at least in one space dimension $[1,2,3]$. Such a description generically maps many-body collective excitations of the fermion system into large coherent bosonic excitations. Some quantum features of the fermion system are therefore mapped to classical features of the bosonic system in a way analogous to duality transformations. Because of this, bosonization is a convenient setting to study strongly correlated systems of fermions.

A particularly intuitive approach to bosonization is a hydrodynamic phase space formulation of the many fermion system, otherwise known as the "droplet" method. In this approach we start with a semiclassical description of the many-body system in terms of a dense collection of particles in their single-particle phase space, forming a constant-density distribution inside a (generically connected) domain, the 'droplet'. The boundary of this domain essentially corresponds to the Fermi surface, and the dynamics of this boundary encodes the many-body degrees of freedom $[4,5]$. In one dimension, this leads to a chiral theory that fully reproduces the standard bosonization results. In particular, for nonrelativistic fermions, it reproduces the results of the collective field method [6]. Fermion excitations around the Fermi (or Dirac) sea become one-dimensional waves with the corresponding phonon states encoding quantum excitations, while properly defined exponentials in the bosonic field ('vertex operators') become fermion operators. Overall, we have a perturbatively exact mapping of states between the two systems. For systems with a finite number of fermions this mapping can fail at the nonperturbative level, when the Fermi sea is completely depleted. For relativistic fermionantifermion systems, the corresponding Dirac sea is bottomless and can never be depleted, so the equivalence is exact even at the nonperturbative level. (An alternative operator approach to bosonization of a finite number of fermions in one space dimension is given in [7].)

The phase space droplet approach works, in principle, in any number of dimensions, at least semiclassically. In fact, an adaptation of this method properly taking into account the quantum nature of the phase space was proposed as the starting point for an exact bosonization in arbitrary dimensions [8]. (For other approaches to higher dimensional bosonization see [9].) A full development of this method and application to realistic, interacting higher dimensional fermion systems remains an interesting project.

The success of the bosonization method relies on the fact that the two systems share the same set of physical states and observables. This means that many-body fermion operators and bosonic operators are into one-to-one correspondence, and the two sets obey the same operator algebra. More importantly, the Hilbert spaces on which these operators act in the two systems are equivalent. This is crucial, as there are examples of systems with the same algebra of observables but with their Hilbert spaces providing inequivalent representations of this algebra. In fact, the albegra of hydrodynamic density operators is such an example, as it admits several representations corresponding to particles with inequivalent (and even exotic) statistics. It is important, therefore, to have a realization of the algebra of observables that admits the many-body fermionic Hilbert space as its (unique and irreducible) representation.

The droplet parametrization achieves just that. Specifically, the phase space density $\hat{\rho}(x, p)$ is a 'universal' operator in terms of which all observables of the many-body system can be expressed, including arbitrary (particle-number preserving) interactions. The realization of 
this operator in terms of the operator representing the boundary of the droplet leads to a representation of the full set of fermion excitations and provides a complete 'dictionary' between the two systems [10].

In this paper we push this method further in the tractable case of one space dimension by deriving the bosonized form of the Hamiltonian for a finite number of fermions for various cases (dispersion relations) that do not seem to have been examined in the literature. This includes the standard results of massless relativistic fermions and nonrelativistic fermions, but generalizes them to arbitrary polynomials or analytic functions of the momentum. Interactions are also considered, demonstrating that the droplet method reveals interesting collective behavior around the Fermi sea. This sets the stage for possible extensions to higher dimensions and for potentially more realistic applications.

\section{Review of the droplet density method}

We consider $N$ fermions in one space dimension. For convenience, we will assume space to be compact and will choose units such that its periodicity be $2 \pi$ (which quantizes single-particle momenta in integer units).

The Weyl-ordered quantum many-body density operator is given by

$$
\hat{\rho}(x, p)=\frac{1}{(2 \pi)^{2}} \sum_{l=-\infty}^{\infty} \int d k \sum_{i=1}^{N} e^{i\left[l\left(\hat{x}_{i}-x\right)+k\left(\hat{p}_{i}-p\right]\right)}
$$

Its Fourier transform $\hat{\rho}(l, k)=\sum_{i=1}^{N} e^{i\left(l \hat{x}_{i}+k \hat{p}_{i}\right)}$ satisfies the well-known "sine" algebra [11]

$$
\left[\hat{\rho}(l, k), \hat{\rho}\left(l^{\prime}, k^{\prime}\right)\right]=-2 i \sin \left(\frac{l k^{\prime}-k l^{\prime}}{2}\right) \hat{\rho}\left(l+l^{\prime}, k+k^{\prime}\right)
$$

Given a single-particle Hamiltonian $H_{s p}(\hat{x}, \hat{p})$, expressed in a Weyl-ordered form in terms of $\hat{x}, \hat{p}$, one can write the exact quantum many-body Hamiltonian in terms of $\hat{\rho}(x, p)$ and $H_{s p}(x, p)$ as

$$
\hat{H}=\sum_{i=1}^{N} H_{s p}\left(\hat{x}_{i}, \hat{p}_{i}\right)=\int H_{s p}(x, p) \hat{\rho}(x, p) d x d p
$$

This can be similarly extended to other quantum operators and also to cases with interactions.

In [10] it was shown that the quantum density operator $\hat{\rho}(x, p)$ can be explicitly written in terms of the boundary operator $\hat{R}(x)$, achieving the bosonization of any many-fermion system and further providing an explicit way of deriving the exact bosonized expressions for all quantum operators of the theory.

The quantum boundary field $\hat{R}(x)$ is a chiral field satisfying the commutation rule

$$
\left[\hat{R}(x), \hat{R}\left(x^{\prime}\right)\right]=-2 \pi i \delta^{\prime}\left(x-x^{\prime}\right)
$$

or in terms of its Fourier modes

$$
\left[\hat{R}_{n}, \hat{R}_{m}\right]=n \delta_{n+m}
$$

where $\hat{R}(x)=\sum_{n=-\infty}^{+\infty} \hat{R}_{n} e^{i n x}$. Semiclassically, it represents the value of the Fermi momentum at position $x$. In fact, there are two such mutually commuting chiral fields, $\hat{R}(x)$ and $\hat{\bar{R}}(x)$, 
corresponding to the two Fermi momenta of a finite particle distribution. Particle excitations around each Fermi momentum actually factorize, so for most purposes considering each section separately will suffice. In the following we will consider only one chiral sector generated by $\hat{R}(x)$. For that purpose we use phase space coordinates $(x, p) \in[-\pi, \pi] \times[0, \infty)$, where the momentum $p$ is positive, essentially considering only "half" the Fermi sea states. We will return to the issue of considering both sectors in the section on interactions.

The Fock space on which the $R_{n}$ 's act consists of the vacuum state $|0\rangle$, which is annihilated by the positive modes $\hat{R}_{n}(n>0)$ and the excited states generated by the action of the negative modes, $\hat{R}_{-n}$, on the vacuum. Since $\hat{R}_{0}$ commutes with all $\hat{R}_{n}$ 's, the action of $\hat{R}_{0}$ on the vacuum defines a conserved quantity $N$, which can be identified with the number of fermions, $\hat{R}_{0}|0\rangle=\frac{N}{2}|0\rangle$. (The remaining half are assigned to the zero mode $\hat{\bar{R}}_{0}$ of the other chiral sector.)

In [10] it was shown that the Fourier transform of the normally ordered quantum density operator can be written in terms of the chiral field $\hat{R}(x)$ as

$$
\hat{\rho}(l, k)=\int \frac{1}{4 \pi i \sin \frac{k}{2}} e^{a(x, k)} e^{b(x, k)} e^{i k \hat{R}_{0}} e^{i l x} d x
$$

where

$$
\begin{aligned}
& a(x, k)=\sum_{n>0} \frac{2 i}{n} \sin \frac{n k}{2} \hat{R}_{-n} e^{-i n x} \\
& b(x, k)=\sum_{n>0} \frac{2 i}{n} \sin \frac{n k}{2} \hat{R}_{n} e^{i n x}
\end{aligned}
$$

The above operators satisfy the commutation relations (2) when acting on the Fock states of $R_{n}$. It is straightforward to show using $(6,7)$ that

$$
\hat{\rho}(x, k)=\frac{1}{2 \pi} \sum_{l=-\infty}^{\infty} \hat{\rho}(l, k) e^{-i l x}=\frac{: e^{i \int_{x-\frac{k}{2}}^{x+\frac{k}{2}} \hat{R}(s) d s}:}{4 \pi i \sin \frac{k}{2}}
$$

The small $k$-limit of the above expression is the Fourier transform of a step function, reproducing the semiclassical droplet result, $\hat{\rho}(x, p)=\frac{1}{2 \pi} \theta(\hat{R}(x)-p)$.

The zero-mode contribution to the density operator is

$$
\rho_{0}(x, p)=\frac{1}{2 \pi} \int_{-\infty}^{\infty} \frac{e^{i k\left(R_{0}-p\right)}}{4 \pi i \sin \frac{k}{2}} d k
$$

Evaluating this using a contour integration and a $k \rightarrow k-i \epsilon$ prescription, we find

$$
\rho_{0}(x, p)=\frac{1}{2 \pi} \theta\left(R_{0}-p\right) \sum_{k} \delta\left(R_{0}-p-\frac{1}{2}-k\right)
$$

For a single-particle Hamiltonian of the form $H_{s p}(x, p)=h(p)$, the zero-point energy for the many-body system is

$$
H_{0}=\int h(p) \rho_{0}(x, p) d x d p=\int_{0}^{R_{0}} h(p) \sum_{k} \delta\left(R_{0}-p-\frac{1}{2}-k\right) d p
$$




$$
=\sum_{n=\epsilon}^{R_{0}-\frac{1}{2}} h(n)
$$

as expected, where $\epsilon=0\left(\frac{1}{2}\right)$ if $N=2 R_{0}$ is odd (even) respectively.

\section{Derivation of Hamiltonians for polynomial dispersion}

Let us first consider the many-body quantum Hamiltonian corresponding to the single-particle Hamiltonian of the form $H_{s p}(x, p)=p^{n}$.

The zero-mode contribution to the energy is (for simplicity we are going to consider $N$ odd)

$$
H_{0}^{(n)}=\int p^{n} \rho_{0}(x, p) d x d p=\sum_{l=0}^{R_{0}-\frac{1}{2}} l^{n}
$$

The sum in (12) has a compact expression in terms of Bernoulli polynomials as follows,

$$
H_{0}^{(n)}=\sum_{l=0}^{R_{0}-\frac{1}{2}} l^{n}=\frac{B_{n+1}\left(R_{0}+\frac{1}{2}\right)-B_{n+1}}{n+1}
$$

where $B_{n+1}(0)=B_{n+1}$ are the corresponding Bernoulli numbers. For large $R_{0}$, the zero-point energy in $(12,13)$ reduces to the semiclassical droplet result $H_{0}^{(n)} \rightarrow R_{0}^{n+1} /(n+1)$.

Using $(8,9)$ we find that the quantum many-body Hamiltonian characterizing the excitations of the system can be written as

$$
\begin{aligned}
H^{(n)}-H_{0}^{(n)} & =\frac{1}{2 \pi} \int p^{n} \frac{: e^{i \int_{x-\frac{k}{2}}^{x+\frac{k}{2}} \hat{R}(s) d s}:-e^{i k R_{0}}}{4 \pi i \sin \frac{k}{2}} e^{-i k p} d k d p d x \\
& =\int_{-\pi}^{\pi} d x\left[\left(\frac{-i \partial_{k}}{2}\right)^{n}\left(\frac{: e^{i \int_{x-k}^{x+k} \hat{R}(s) d s}:-e^{2 i k R_{0}}}{4 \pi i \sin k}\right)\right]_{k=0}
\end{aligned}
$$

The exponential term containing the boundary field $R(s)$ can be written as a series expansion in terms of $R(x)$ and its even derivatives as (we drop hats from operators from now on)

$: \exp \left(i \int_{x-k}^{x+k} R(s) d s\right):=: \exp \left(2 i k\left[R(x)+\sum_{l=1} k^{2 l} \frac{\partial_{x}^{2 l} R(x)}{(2 l+1) !}\right]\right) \equiv: \exp \left(2 i k\left(R(x)+R_{D}(k, x)\right)\right):$

where the term $R_{D}(k, x)$ contains only derivatives of the field $R(x)$. As a result, the Hamiltonian in (14) can be written as an integral of a polynomial given in terms of the boundary field $R(x)$ and its even derivatives.

Using (15), we can write the expression for the Hamiltonian in (14) as

$$
H^{(n)}-H_{0}^{(n)}=\left(-\frac{i}{2}\right)^{n} \int_{-\pi}^{\pi} d x\left(\partial_{k}\right)^{n}\left[: \frac{e^{2 i k R(x)}-e^{2 i k R_{0}}}{4 \pi i \sin k}+\frac{e^{2 i k\left(R(x)+R_{D}(x, k)\right)}-e^{2 i k R(x)}}{4 \pi i \sin k}:\right]_{k=0}
$$


The first term in (16) is a polynomial in $R(x)$ (does not contain any derivatives of the field), while the second term is an expression containing even derivatives of the field. We shall now calculate these two terms separately. A useful formula for these calculations is the one giving the generating function for Bernoulli polynomials, namely

$$
\frac{t e^{z t}}{e^{t}-1}=\sum_{n=0}^{\infty} B_{n}(z) \frac{t^{n}}{n !}
$$

Using (17) we find

$$
: \frac{e^{2 i k R(x)}-e^{2 i k R_{0}}}{\sin k}:=2 i \sum_{l=0}^{\infty}: \frac{\left[B_{l+1}\left(R+\frac{1}{2}\right)-B_{l+1}\left(R_{0}+\frac{1}{2}\right)\right]}{l+1}: \frac{(2 i k)^{l}}{l !}
$$

where we used the fact that $B_{0}(z)=1$ for any $z$. Using (18) in (16) we easily find that

$$
H^{(n)}-H_{0}^{(n)}=\frac{1}{2 \pi} \int d x\left[: \frac{B_{n+1}\left(R(x)+\frac{1}{2}\right)-B_{n+1}\left(R_{0}+\frac{1}{2}\right)}{n+1}+(\text { derivative terms }):\right]
$$

Further using (12) we find that

$$
H^{(n)}=\frac{1}{2 \pi} \int d x\left[: \frac{B_{n+1}\left(R(x)+\frac{1}{2}\right)-B_{n+1}}{n+1}+(\text { derivative terms }):\right]
$$

We now outline the calculation of the derivative terms in (20) which is somewhat more involved. Using the following relation for Bernoulli polynomials

$$
B_{s}(x+y)=\sum_{j=0}^{s} \frac{s !}{j !(s-j) !} y^{j} B_{s-j}(x)
$$

and (17) we find that

$$
: \frac{e^{2 i k\left(R(x)+R_{D}(x, k)\right)}-e^{2 i k R(x)}}{\sin k}:=\sum_{s=1}^{\infty} \sum_{j=1}^{s} \frac{1}{j !(s-j) !}: B_{s-j}\left(R+\frac{1}{2}\right)\left(R_{D}\right)^{j}:(2 i k)^{s-1}
$$

where $\left(R_{D}\right)^{j}$ can be expanded in terms of even derivatives of $R(x)$ as

$$
\begin{aligned}
\left(R_{D}(k, x)\right)^{j} & =\left(\sum_{i=1}^{\infty} k^{2 i} d_{2 i}\right)^{j}=\sum_{r_{1}+\cdots r_{i}=j} \frac{j !}{r_{1} ! r_{2} ! \cdots r_{i} !} k^{2 r_{1}+4 r_{2}+\cdots 2 i r_{i}} d_{2}^{r_{1}} d_{4}^{r_{2}} \cdots d_{2 i}^{r_{i}} \\
d_{2 i} & \equiv \frac{\partial_{x}^{2 i} R(x)}{(2 i+1) !}
\end{aligned}
$$

Defining the terms in the Hamiltonian (20) that depend on the derivatives of the field $R(x)$ as $H_{D}^{(n)}$ and using $(22,23)$ we find

$$
H_{D}^{(n)}=\frac{1}{2 \pi} \int d x: \sum_{l=1}^{\{n / 2\}} \sum_{j=1}^{l} \frac{(-1)^{l}}{2^{2 l}} \frac{n ! B_{n+1-2 l-j}\left(R+\frac{1}{2}\right)}{(n+1-2 l-j) !} \sum_{\substack{r_{1}+\cdots r_{i}=j, r_{1}+2 r_{2}+\cdots+i r_{i}=l}} \frac{d_{2}^{r_{1}} d_{4}^{r_{2}} \cdots d_{2 i}^{r_{i}}}{r_{1} ! r_{2} ! \cdots r_{i} !}:
$$


where $\{n / 2\}=n / 2$ if $n$ is even ( $=\frac{n-1}{2}$ if $n$ is odd). This gives an expansion in terms of the total number of derivatives.

Expressions $(20,24)$ give the exact quantum many-body Hamiltonian for a fermionic system with single particle Hamiltonian of the form $p^{n}$ in terms of the boundary field $R(x)$ and its derivatives. Here are the results for $H^{(n)}, n=1, \cdots 5$ :

$\underline{n=1}$

$$
H^{(1)}=\frac{1}{2 \pi} \int d x: \frac{B_{2}\left(R+\frac{1}{2}\right)-B_{2}}{2}:=\frac{1}{2 \pi} \int d x:\left(\frac{R^{2}}{2}-\frac{1}{8}\right):
$$

$\underline{n=2}$

$$
H^{(2)}=\frac{1}{2 \pi} \int d x: \frac{B_{3}\left(R+\frac{1}{2}\right)-B_{3}}{3}:=\frac{1}{2 \pi} \int d x:\left(\frac{R^{3}}{3}-\frac{R}{12}\right):
$$

$\underline{n=3}$

$$
\begin{aligned}
H^{(3)} & =\frac{1}{2 \pi} \int d x: \frac{B_{4}\left(R+\frac{1}{2}\right)-B_{4}}{4}-\frac{B_{1}\left(R+\frac{1}{2}\right)}{4} R^{\prime \prime}(x): \\
& =\frac{1}{2 \pi} \int d x:\left(\frac{\left(R^{2}-\frac{1}{4}\right)^{2}}{4}-\frac{R R^{\prime \prime}}{4}\right):
\end{aligned}
$$

$\underline{n=4}$

$$
\begin{aligned}
H^{(4)} & =\frac{1}{2 \pi} \int d x: \frac{B_{5}\left(R+\frac{1}{2}\right)-B_{5}}{5}-\frac{1}{2} B_{2}\left(R+\frac{1}{2}\right) R^{\prime \prime}(x): \\
& =\frac{1}{2 \pi} \int d x:\left(\frac{R^{5}}{5}-\frac{R^{3}}{6}+\frac{7 R}{240}-\frac{R^{2} R^{\prime \prime}}{2}\right):
\end{aligned}
$$

$\underline{n=5}$

$$
\begin{aligned}
H^{(5)} & =\frac{1}{2 \pi} \int d x: \frac{B_{6}\left(R+\frac{1}{2}\right)-B_{6}}{5}-\frac{5}{6} B_{3}\left(R+\frac{1}{2}\right) R^{\prime \prime}(x)+\frac{1}{16} B_{1}\left(R+\frac{1}{2}\right) R^{\prime \prime \prime \prime}(x)+\frac{5}{48}\left(R^{\prime \prime}\right)^{2}: \\
& =\frac{1}{2 \pi} \int d x:\left(\frac{R^{6}}{6}-\frac{5 R^{4}}{24}+\frac{7 R^{2}}{96}-\frac{1}{128}-\frac{5}{6}\left(R^{3}-\frac{R}{4}\right) R^{\prime \prime}+\frac{R R^{\prime \prime \prime \prime}}{16}+\frac{5\left(R^{\prime \prime}\right)^{2}}{48}\right):
\end{aligned}
$$

In all the above expressions we have neglected total derivative terms.

\section{Extension to arbitrary functions of the momentum}

The calculation of the many-body quantum Hamiltonian can now be extended to cases where the single-particle Hamiltonian is an arbitrary function of momentum, namely $H_{s p}=f(p)$. 
Suppose that $f(p)$ admits a Taylor series expansion

$$
f(p)=\sum_{n=0}^{\infty} \frac{p^{n}}{n !} \partial^{n} f(0)
$$

Then

$$
H=\sum_{n=0}^{\infty} \frac{\partial^{n} f(0)}{n !} \int p^{n} \hat{\rho}(x, p) d x d p=\sum_{n=0}^{\infty} \frac{\partial^{n} f(0)}{n !} H^{(n)}
$$

For simplicity we shall split the calculation of $H$ into two terms: $H_{N D}$ will be the contribution from the non-derivative part of $H^{(n)}$, given in (20), and $H_{D}$ will be the contribution from the derivative part $H_{D}^{(n)}$, given in (24):

$$
\begin{aligned}
H & =H_{N D}+H_{D} \\
H_{N D} & =\frac{1}{2 \pi} \int d x \sum_{n=0}^{\infty} \frac{\partial^{n} f(0)}{n !}: \frac{B_{n+1}\left(R(x)+\frac{1}{2}\right)-B_{n+1}}{n+1}: \\
H_{D} & =\frac{1}{2 \pi} \int d x \sum_{n=0}^{\infty} \frac{\partial^{n} f(0)}{n !}: \sum_{l=1}^{\{n / 2\}} \sum_{j=1}^{l} \frac{(-1)^{l}}{2^{2 l}} \frac{n ! B_{n+1-2 l-j}\left(R+\frac{1}{2}\right)}{(n+1-2 l-j) !} \sum_{\substack{r_{1}+\cdots r_{i}=j, r_{1}+\cdots+i r_{i}=l}} \frac{d_{2}^{r_{1}} d_{4}^{r_{2}} \cdots d_{2 i}^{r_{i}}}{r_{1} ! r_{2} ! \cdots r_{i} !}:
\end{aligned}
$$

Using the following relation for Bernoulli polynomials

$$
\int_{a}^{x} B_{r}(t)=\frac{B_{r+1}(x)-B_{r+1}(a)}{r+1}
$$

we can write

$$
H_{N D}=\frac{1}{2 \pi} \int d x: \int_{0}^{R+\frac{1}{2}} \sum_{n=0}^{\infty} \frac{B_{n}(t)}{n !} \partial^{n} f(0):
$$

Further the Bernoulli polynomial can be expanded in terms of Bernoulli numbers as

$$
B_{n}(t)=\sum_{k=0}^{n} \frac{B_{k}}{k !} \frac{n !}{(n-k) !} t^{n-k}=\sum_{k=0}^{n} \frac{B_{k}}{k !}\left(\partial_{t}\right)^{k} t^{n}
$$

Using $z=0$ and replacing $t \rightarrow \partial_{t}$ in (17) one can easily show that

$$
B_{n}(t)=\frac{\partial}{e^{\partial}-1} t^{n}
$$

Using this in (25) we find that we can express $H_{N D}$ as

$$
H_{N D}=\frac{1}{2 \pi} \int d x: \int_{0}^{R+\frac{1}{2}} d t \frac{\partial}{e^{\partial}-1} f(t):
$$

If we define $G(t)=\frac{\partial}{e^{\partial}-1} f(t)$ then $G(t)$ has to satisfy the relation $G(t+1)-G(t)=\partial f(t)$. For special cases of $f(t), G(t)$ has a compact form. For example,

$$
f(t)=t^{n} \Longrightarrow G(t)=B_{n}(t)
$$




$$
f(t)=e^{a t} \Longrightarrow G(t)=\frac{e^{a t}}{e^{a}-1}
$$

In general though,

$$
G(t)=\frac{\partial}{e^{\partial}-1} f(t)=f(t)+B_{1} \partial f(t)+\sum_{k=1}^{\infty} \frac{B_{2 k}}{(2 k) !} \partial^{2 k} f(t)
$$

Using this in equation (37) we find

$$
\begin{aligned}
H_{N D} & =\int \frac{d x}{2 \pi}:\left[\int_{0}^{R+\frac{1}{2}} d t f(t)-\frac{1}{2}\left(f\left(R+\frac{1}{2}\right)-f(0)\right)+\sum_{k=1}^{\infty} \frac{B_{2 k}}{(2 k) !}\left[\left.\partial_{u}^{2 k-1} f(u)\right|_{u=R+\frac{1}{2}}-\left.\partial_{u}^{2 k-1} f(u)\right|_{u=0}\right]\right]: \\
& \equiv \int \frac{d x}{2 \pi}: F\left(R(x)+\frac{1}{2}\right):
\end{aligned}
$$

where we used the fact that $B_{1}=-1 / 2$. The expression (39) is not surprising. When $H_{N D}$ acts on the vacuum state, $R+\frac{1}{2}$ is replaced by $(N+1) / 2$ which is an integer (we have assumed $N$ to be odd). In this case (39) is just the Euler-MacLauren formula connecting a sum with an integral, namely

$$
\begin{aligned}
E & =\sum_{i=0}^{\frac{N-1}{2}} f(i) \\
& =\int_{0}^{\frac{N+1}{2}} d t f(t)-\frac{1}{2}\left(f\left(\frac{N+1}{2}\right)-f(0)\right)+\sum_{k=1}^{\infty} \frac{B_{2 k}}{(2 k) !}\left[\left.\partial_{u}^{2 k-1} f(u)\right|_{u=\frac{N+1}{2}}-\left.\partial_{u}^{2 k-1} f(u)\right|_{u=0}\right]
\end{aligned}
$$

as expected. One can view the operator expression : $F\left(R(x)+\frac{1}{2}\right):$ in (39) as the "operator" definition of (41) when $R_{0}=N / 2$ is replaced by the field $R(x)$.

Let us now evaluate the part of the Hamiltonian that contains derivatives of the boundary field. We found in (24) that the $2 l$-derivative term in the $H^{(n)}$ case $(n \geq 2 l)$ is of the form

$$
H_{D^{2 l}}^{(n)}=\frac{1}{2 \pi} \int d x: \sum_{j=1}^{l} \frac{(-1)^{l}}{2^{2 l}} \frac{n ! B_{n+1-2 l-j}\left(R+\frac{1}{2}\right)}{(n+1-2 l-j) !} \sum_{\substack{r_{1}+\cdots r_{i}=j, r_{1}+2 r_{2}+\cdots+i r_{i}=l}} \frac{d_{2}^{r_{1}} d_{4}^{r_{2}} \cdots d_{2 i}^{r_{i}}}{r_{1} ! r_{2} ! \cdots r_{i} !}:
$$

Using the following property of the Bernoulli polynomials

$$
\partial_{t}^{i} B_{n}(t)=\frac{n !}{(n-i) !} B_{n-i}(t) \quad, \quad n \geq i
$$

as well as equations (35-41) one can show that the $2 l$-derivative part of $H_{D}$ in (32) can be written as

$$
H_{D^{2 l}}=\frac{1}{2 \pi} \int d x:\left.\sum_{j=1}^{l} \frac{(-1)^{l}}{2^{2 l}} \sum_{\begin{array}{c}
r_{1}+\cdots r_{i}=j, \\
r_{1}+2 r_{2}+\cdots+i r_{i}=l
\end{array}} \frac{d_{2}^{r_{1}} d_{4}^{r_{2}} \cdots d_{2 i}^{r_{i}}}{r_{1} ! r_{2} ! \cdots r_{i} !} \partial_{u}^{2 l+j} F(u)\right|_{u=R+\frac{1}{2}}:
$$


Combining the non-derivative term, eq. (39), and the derivative terms, eq.(43), we find that the full Hamiltonian can be written as

$$
H=\frac{1}{2 \pi} \int d x:\left.\exp \left(\sum_{i=1}^{\infty} \frac{(-1)^{i} \partial_{x}^{2 i} R(x)}{2^{2 i}(2 i+1) !} \partial_{u}^{2 i+1}\right) F(u)\right|_{u=R+\frac{1}{2}} \quad:
$$

where $F\left(R+\frac{1}{2}\right)$ is defined in $(39)$.

In the case where the single particle Hamiltonian is polynomial in momentum, $f(p)=p^{n}$, we have

$$
F\left(R+\frac{1}{2}\right)=\int_{0}^{R+\frac{1}{2}} d t B_{n}(t)=\frac{B_{n+1}\left(R(x)+\frac{1}{2}\right)-B_{n+1}}{n+1}
$$

and the corresponding expression (44) provides a compact expression for (20, 24).

An interesting application of (44) is in the case of noninteracting massive relativistic fermions, where $f(p)=\sqrt{p^{2}+m^{2}}$. (We consider them spinless, or assume spin to be 'frozen' in one direction.) In this case $F\left(R+\frac{1}{2}\right)$, as defined in (39), has an infinite series expansion in $1 / N$. Keeping up to $\mathcal{O}(1)$ terms, we find

$$
H=\int \frac{d x}{2 \pi}: F\left(R(x)+\frac{1}{2}\right):
$$

where

$$
\begin{aligned}
F\left(R+\frac{1}{2}\right)= & \frac{1}{2}\left(R+\frac{1}{2}\right) \sqrt{m^{2}+\left(R+\frac{1}{2}\right)^{2}}+\frac{m^{2}}{2} \ln \left(\frac{R+\frac{1}{2}}{m}+\sqrt{1+\frac{\left(R+\frac{1}{2}\right)^{2}}{m^{2}}}\right) \\
& -\frac{1}{2}\left(\sqrt{m^{2}+\left(R+\frac{1}{2}\right)^{2}}-m\right)+\frac{1}{12}\left(\frac{R+\frac{1}{2}}{\sqrt{m^{2}+\left(R+\frac{1}{2}\right)^{2}}}-\frac{\epsilon}{\sqrt{m^{2}+\epsilon^{2}}}\right)
\end{aligned}
$$

where $\epsilon \rightarrow 0$ eventually. (Keeping $\epsilon$ finite is important in considering the $m \rightarrow 0$ limit.) Terms containing derivatives of the boundary field are $\mathcal{O}(1 / N)$ and are neglected in (47).

The $m \rightarrow 0$ of the Hamiltonian in (47) reproduces the expression for massless relativistic particle in $(25)$.

\section{$5 \quad$ Interactions}

The droplet formalism allows for the introduction of interactions in a straightforward way. We shall consider space-dependent interactions only, and focus on one-body and two-body potentials.

The important element when including interactions is that, in general, we may not consider each chiral sector of the theory separately. Indeed, the space particle density operator

$$
\rho(x)=\int d p \rho(x, p), \quad\left[\rho(x), \rho\left(x^{\prime}\right)\right]=0
$$


would not even commute with itself if only one sector were included. We thus consider the two boundary operators $R(x)$ and $\bar{R}(x)$ corresponding to the two Fermi momenta in semiclassical phase space. The opposite-chirality boundary field $\bar{R}$ satisfies

$$
\left[\bar{R}(x), \bar{R}\left(x^{\prime}\right)\right]=+2 \pi i \delta^{\prime}\left(x-x^{\prime}\right)
$$

with a crucial opposite sign in the commutator. The modes are, then, defined with a corresponding negative sign

$$
\bar{R}(x)=\sum_{n=-\infty}^{\infty} \bar{R}_{n} e^{-i n x}
$$

so that positive (negative) $n$ still label annihilation (creation) operators. The modes satisfy

$$
\left[\bar{R}_{n}, \bar{R}_{m}\right]=n \delta_{n+m}, \quad\left[\bar{R}_{n}, R_{m}\right]=0
$$

All corresponding many-boby operators, such as the density operator and the Hamiltonian, become the difference of two expressions involving $R$ and $\bar{R}$. The space density, in particular, becomes

$$
\rho(x)=\frac{1}{2 \pi}[R(x)-\bar{R}(x)]
$$

and in terms of modes

$$
\rho_{n}=\frac{1}{2 \pi}\left(R_{n}-\bar{R}_{-n}\right)
$$

We see that the $\rho_{n}$ commute among themselves. The total number of particles is

$$
N=2 \pi \rho_{0}=R_{0}-\bar{R}_{0}
$$

and in a symmetric situation $R_{0}=-\bar{R}_{0}=\frac{N}{2}$.

\subsection{One-body potentials}

In the presence of a one-body potentail $V(x)$ the Hamiltonian aquires the extra term

$$
U=\int d x V(x) \rho(x)=\sum_{n=-\infty}^{\infty} V_{-n} R_{n}-\sum_{n=-\infty}^{\infty} V_{n} \bar{R}_{n}
$$

and the full Hamiltonian, consisting of a general momentum term as analyzed in the previous sections and the potential term, becomes

$$
H_{i n t}=H[R]+\int d x V(x) R(x)-H[\bar{R}]-\int d x V(-x) \bar{R}(x)
$$

with $H[R]$ the free Hamiltonian as given in (44). We see that in the case of one-body potentials the full Hamiltonian remains the sum of two chiral terms, so the two sectors do not mix and can be considered separately.

To explore the spectrum of the above Hamiltonian, we will work to leading order in $1 / N$. All the derivative terms in (44) are subleading, so we get for the $R$-chirality sector

$$
H=\frac{1}{2 \pi} \int d x: F_{o}(R(x))+V(x) R(x):
$$


where we defined $F_{o}(R)=F\left(R+\frac{1}{2}\right)$. Note that for a 'relativistic' single-particle Hamiltonian linear in momentum ( $F_{o}$ quadratic) the leading $1 / N$ approximation is (perturbatively) exact.

We proceed to linearize and diagonalize the Hamiltonian by considering the solution $R_{c}(x)$ to the classical equation

$$
F_{o}^{\prime}\left(R_{c}(x)\right)+V(x)=E_{F}
$$

$E_{F}$, which can be thought of as the "chemical potential" Lagrange multiplier enforcing the constraint $R_{0}-\bar{R}_{0}=N$, is essentially the semiclassical Fermi level energy. It is determined by the requirement that the solution of the above equation satisfy

$$
\frac{1}{2 \pi} \int d x R_{c}(x)=\frac{N}{2}
$$

assuming similar equations for $\bar{R}$ and a symmetric situation. Writing

$$
R(x)=R_{c}(x)+r(x)
$$

the new operator $r(x)$ satisfies the same chiral boundary algebra as $R(x)$ as it is shifted by a c-number term. It terms of $r(x)$ the Hamiltonian becomes (dropping explicit $x$-dependence)

$$
H=\frac{1}{2 \pi} \int d x:\left\{F_{o}\left(R_{c}\right)+V R_{c}+\left[F_{o}^{\prime}\left(R_{c}\right)+V\right] r+\frac{1}{2} F_{o}^{\prime \prime}\left(R_{c}\right) r^{2}+O\left(r^{3}\right)\right\}:
$$

Due to the equation (58) the term in the middle bracket is a constant and it contributes to the Hamiltonian a term proportional to $E_{F} r_{0}$. The zero mode of $r$ corresponds to additional particles at this Fermi level. Since $R_{0}-\bar{R}_{0}=N$, any additional particle appearing in $R_{0}$ would have to come by migrating from the opposite Fermi level $\bar{R}_{0}$. Such moves across Fermi levels constitute nonperturbative excitations and, at any rate, can be accounted for separately as $r_{0}=\bar{r}_{0}$ are Casimirs and commute with all other operators. Omitting this term, then, the Hamiltonian to leading order in $1 / N$ (quadratic in $r$ ) becomes

$$
H=E_{o}+\frac{1}{2 \pi} \int d x \frac{1}{2}: v r^{2}:
$$

with $v(x)=F_{o}^{\prime \prime}\left(R_{c}(x)\right)$ the semiclassical Fermi velocity and $E_{o}$ the constant term in (61). We define a new coordinate $y$ by

$$
\frac{d x}{v(x)}=\alpha d y, \quad 2 \pi \alpha=\int \frac{d x}{v(x)}
$$

with the coefficient $\alpha$ chosen so that $y$ have a periodicity of $2 \pi$. We also define the new operator $\phi=v r$ whose Fourier modes in the $y$ variable are

$$
\phi_{n}=\frac{1}{2 \pi} \int d y \phi(y) e^{i n y}=\frac{1}{2 \pi} \int d x r(x) e^{i n y(x)}
$$

and satisfy the standard chiral algebra

$$
\left[\phi_{n}, \phi_{m}\right]=n \delta_{n+m}
$$


In terms of the above, the Hamiltonian becomes (omitting the constant $E_{o}$ )

$$
H=\frac{1}{2 \pi} \int d y \frac{1}{2} \alpha: \phi(y)^{2}:=\alpha \sum_{n>0}^{\infty} \phi_{-n} \phi_{n}
$$

This is a set of decoupled harmonic oscillators. The normal ordering in the new field obviously has to be done in terms of the modes $\phi_{n}$ leading to a positive definite Hamiltonian. The excitations are equidistant over the ground state with a spacing $\alpha$ between the single-particle energy levels.

The above results can be recovered in the many-body language through a semiclassical calculation. The new coordinate $y$ is essentially the time of flight of a particle at the Fermi energy $E_{F}$ and $\alpha$ is the single-particle energy spectrum spacing near the Fermi level as derived in a WKB approximation. What the above calculation shows is that the WKB result becomes exact in the large- $N$ limit, and it is exact to all orders in $1 / N$ for a single-body kinetic term linear in the momentum.

Using the above formalism we can also keep higher order terms in $r$ and $1 / N$ and calculate corrections to the many-body energy spectrum. In this case, $F_{o}(R)$ in $(57)$ has to be modified by keeping the appropriate subleading derivative terms appearing in (44). For arbitrary potentials, however, this becomes a rather complicated calculation.

\subsection{Two-body potentials}

The situation becomes a lot more interesting when we consider two-body potentials. The two chiral sectors mix, and genuine collective effects come into play.

We will consider translationally invariant, symmetric two-body potentials of the form

$$
U=\sum_{i<j} V\left(x_{i}-x_{j}\right)
$$

with $V(x)$ an even real function of $x$. We will also assume that $V(x)$ is regular at $x=0$, so no singularities arise as two particles coincide. The case of singular potentials, such as the Coulomb $\left(V \sim|x|^{-1}\right)$ or the "Calogero" $\left(V \sim x^{-2}\right)$ potential, require a more careful treatment.

The potential energy can be expressed in terms of the particle density operator $\rho(x)$ as

$$
U=\frac{1}{2}\left[\int d x d y V(x-y) \rho(x) \rho(y)-\int d x V(0) \rho(x)\right]
$$

where the second term removes the particle self-interactions (the terms $i=j$ in the many-body sum). Expressing $V(x)$ in terms of its Fourier modes

$$
V(x)=\sum_{n} V_{n} e^{i n x}
$$

with $V_{-n}=V_{n}$ real, the above expression becomes

$$
U=(2 \pi)^{2} \sum_{n>0} V_{n} \rho_{-n} \rho_{n}+\frac{1}{2}(2 \pi)^{2} V_{0} \rho_{0}^{2}-\pi V(0) \rho_{0}
$$


Expressing the density in terms of chiral modes as in (53) and assuming again a symmetric situation in which $R_{0}=-\bar{R}_{0}=\frac{N}{2}$ we obtain

$$
U=\frac{1}{2} V_{0} N(N-1)+\sum_{n>0}(n-N) V_{n}+\sum_{n>0} V_{n}\left(R_{-n} R_{n}+\bar{R}_{-n} \bar{R}_{n}-R_{n} \bar{R}_{n}-R_{-n} \bar{R}_{-n}\right)
$$

where we also used $V(0)=V_{0}+2 \sum_{n>0} V_{n}$.

Before proceeding to the full Hamiltonian, we comment on the validity range of the above expression for the interaction energy. As stated earlier, droplet bosonization results are perturbatively exact, up to the point that large excitations deplete the Fermi sea and mix the two chiral sectors. The Fourier mode $V_{n}$ of the interaction potential, on the other hand, generically creates excitations of order $n$ in the fermion state. To prevent the above-mentioned nonperturbative effects, $n$ should be of order smaller than $N$. Therefore, only smooth enough potentials, with vanishing Fourier modes as $n$ becomes of order $N$, are reliably expressed in the droplet bosonization formula (71).

It is instructive to consider the special case of a delta-function two-body potential

$$
V(x)=c \delta(x), \quad V_{n}=\frac{c}{2 \pi}
$$

This potential is special in that it clearly violates the condition stated above, as it has nonvanishing modes for all $n$, and in that it is actually irrelevant for fermions. Indeed, its support is only at particle coincidence points where the fermionic wavefunction vanishes. Therefore, its expectation value for any fermionic state should vanish.

Applying formula (71) for $V_{n}=c / 2 \pi$ and for the free fermion homogeneous ground state annihilated by all $R_{n}$ and $\bar{R}_{n}, n>0$, the expectation value of the operator term vanishes. The constant terms give a formally infinite result due to the infinite sum in $V_{n}$. The distance between the two Fermi levels in this state, however, equals $N-1$, so only modes with $n$ up to $N-1$ should be considered. Truncating the sum over such modes we have

$$
\langle U\rangle=\frac{1}{2} \frac{c}{2 \pi} N(N-1)+\sum_{n=1}^{N-1}(n-N) \frac{c}{2 \pi}=0
$$

as required. For excited states, the operator part would also contribute. For such states, modes with $n$ less than $N-1$ will also deplete the Fermi sea and mix the two chiral sectors. The issue of projecting the Fock states of the droplet oscillators to the proper Hilbert state of the fermion system is quite nontrivial and will not be treated here.

We now proceed to deriving the full Hamiltonian and its spectrum. In the absence of any potentials, the (free) ground state is the homogeneous ground state of all oscillators $R_{n}$ and $\bar{R}_{n}$. We will focus again to a symmetric situation and expand around the constant background

$$
R_{c}=-\bar{R}_{c}=\frac{N}{2}
$$

which amounts to setting the zero mode of the chiral fields to $N / 2$. Working, again, to leading order in $1 / N$, the free Hamiltonian will be

$$
H_{o}=K_{o}+\frac{1}{2 \pi} \int d x \frac{1}{2} v: R^{2}+\bar{R}^{2}:
$$


with $K_{o}$ the ground state kinetic energy and $v$ the Fermi velocity, which is constant and common to both sectors:

$$
K_{o}=2 F_{o}\left(\frac{N}{2}\right), \quad v=F_{o}^{\prime \prime}\left(\frac{N}{2}\right)
$$

The total Hamiltonian $H_{o}+U$ expressed in modes becomes

$$
H=E_{o}+\sum_{n>0}\left(v+V_{n}\right)\left(R_{-n} R_{n}+\bar{R}_{-n} \bar{R}_{n}\right)-\sum_{n>0} V_{n}\left(R_{n} \bar{R}_{n}+R_{-n} \bar{R}_{-n}\right)
$$

with the constant term being

$$
E_{o}=2 F_{o}\left(\frac{N}{2}\right)+\frac{1}{2} V_{0} N(N-1)+\sum_{n>0}(n-N) V_{n}
$$

We see that the two chiral sectors mix, but individual modes decouple. We focus, therefore, on the operator part of the Hamiltonian for a single mode $n$. Each such term can be brought to standard oscillator form through a Bogoliubov transformation. To do that, we first decouple the two sectors by performing the redefinitions

$$
A_{n}=\frac{1}{\sqrt{2}}\left(R_{n}+\bar{R}_{n}\right), \quad B_{n}=\frac{1}{\sqrt{2}}\left(R_{n}-\bar{R}_{n}\right)
$$

The Hamiltonian for mode $n$ becomes

$$
H_{n}=\left(v+V_{n}\right) A_{-n} A_{n}-\frac{1}{2} V_{n}\left(A_{n}^{2}+A_{-n}^{2}\right)+\left(v+V_{n}\right) B_{-n} B_{n}+\frac{1}{2} V_{n}\left(B_{n}^{2}+B_{-n}^{2}\right)
$$

The $A$ and $B$ parts are now decoupled and essentially identical in form up to a sign (which can be absorbed by the redefinition $\left.B_{n} \rightarrow i \operatorname{sgn}(n) B_{n}\right)$. The $A$ part can be diagonalized in the standard way by defining the new oscillator operators

$$
a_{n}=\operatorname{ch} \theta A_{n}-\operatorname{sh} \theta A_{-n}
$$

The new $a_{n}$ obey the same algebra as $R_{n}$. With the choice of the parameter $\theta$

$$
\operatorname{th} 2 \theta=\frac{V_{n}}{v+V_{n}} \quad \text { or } \quad \operatorname{ch} \theta=\sqrt{\frac{v+V_{n}}{2 \sqrt{v\left(v+2 V_{n}\right)}}+\frac{1}{2}}
$$

the $A$ part of the Hamiltonian takes the form

$$
H\left(a_{n}\right)=\sqrt{v\left(v+2 V_{n}\right)} a_{-n} a_{n}-\frac{n}{2}\left(v+V_{n}-\sqrt{v\left(v+2 V_{n}\right)}\right)
$$

The $B$ part of the Hamiltonian can be diagonalized with a similar transformation involving the opposite parameter $\theta$ :

$$
b_{n}=\operatorname{ch} \theta B_{n}+\operatorname{sh} \theta B_{-n}
$$

Putting everything together we obtain the final, decoupled, normal form for the full Hamiltonian

$$
H=E_{G}+\sum_{n>0} \sqrt{v\left(v+2 V_{n}\right)}\left(a_{-n} a_{n}+b_{-n} b_{n}\right)
$$


with the ground state energy

$$
E_{G}=2 F_{o}\left(\frac{N}{2}\right)+\frac{1}{2} V_{0} N(N-1)+\sum_{n>0}\left[n\left(\sqrt{v^{2}+2 v V_{n}}-v\right)-N V_{n}\right]
$$

The ground state is annihilated by the operators $a_{n}$ and $b_{n}, n>0$, but it is actually a "squeezed state" in terms of the old oscillators $R_{n}$ and $\bar{R}_{n}$. Excited states are build as Fock states of the oscillators $a_{n}$ and $b_{n}$, but with a renormalized energy gap $\sqrt{v\left(v+2 V_{n}\right)}$ instead of $v$. The gaps depend on $V_{n}$ and are generically different for different modes, so the degeneracy of the noninteracting particle spectrum is lifted. Interestingly, the Hamiltonian is still the sum of two commuting pseudo-chiral sectors, although each sector is really a mixture of the two chiral modes.

\subsection{Phase transition}

From the relation (82) it is clear that the transformation leading to the above Hamiltonian (85) is valid only if

$$
\left|\frac{V_{n}}{v+V_{n}}\right|<1 \quad \text { or } \quad V_{n}>-\frac{v}{2}
$$

For $V_{n}$ below the above critical value the Hamiltonian becomes unbounded from below. The homogeneous state over which this Hamiltonian was constructed becomes unstable, signaling a phase transition. In this case, higher order terms in $1 / N$ (cubic and higher order in $R$ and $\bar{R}$ ) and nonperturbative effects cannot be neglected any more. The true ground state will involve lumping of particles together.

To gain some intuitive understanding of the above phase transition we consider the case of regular nonrelativistic fermions of unit mass and a two-body potential with a single nonzero Fourier mode $V_{1}$. The Fermi velocity is the same as the Fermi momentum

$$
v=R_{0}=\frac{N}{2}
$$

and the potential is

$$
V(x)=2 V_{1} \cos x \simeq 2 V_{1}-V_{1} x^{2}+\cdots
$$

This potential is repulsive for $V_{1}>0$, so clearly it cannot induce any particle lumping and phase transitions, but it is attractive for $V_{1}<0$. For small particle separation it becomes a mutual harmonic oscillator attraction. Setting $V_{1}=-k / 2$ for positive $k$, we can approximate the many-body Hamiltonian for small separations as

$$
H=\sum_{i} \frac{1}{2} p_{i}^{2}+\sum_{i<j} \frac{1}{2} k\left(x_{i}-x_{j}\right)^{2}=\sum_{i} \frac{1}{2} p_{i}^{2}+\sum_{i} \frac{1}{2} N k x_{i}^{2}-\frac{1}{2} k\left(\sum_{i} x_{i}\right)^{2}
$$

So we have essentially $N$ fermions in an external harmonic potential $\frac{1}{2} N k x^{2}$ but with the center of mass oscillator energy removed. The zero-momentum ground state is

$$
\Psi_{0}=C \prod_{i<j}\left(x_{i}-x_{j}\right) e^{-\frac{1}{2} \sqrt{N k}\left[\sum_{i} x_{i}^{2}-\frac{1}{N}\left(\sum_{i} x_{i}\right)^{2}\right]}
$$


with $C$ a normalization constant. To leading order in $1 / N$ this is the wavefunction of $N$ noninteracting fermions in a harmonic trap. The density distribution in the ground state is the well-known Wigner semicircle

$$
\rho_{0}(x)=\frac{\sqrt{N k}}{\pi} \sqrt{a^{2}-x^{2}}, \quad a=\left(\frac{4 N}{k}\right)^{\frac{1}{4}}
$$

As long as the radius $a$ of the semicircle is much smaller than the length $2 \pi$ of the periodic space in which fermions live, the ground state will be well approximated by the above wavefunction and fermions will stay close to each other. That is, for particle separations larger than $2 a$ the fermion two-point function will vanish. This is the "lumped" phase.

However, when the size of the semicircle becomes comparable to or larger than the size of space, its ends will start 'touching'. Further, the quadratic approximation of the potential will not be adequate any more. The fermions will assume a ground state that fills the available space with a nonzero two-point function everywhere. This is the "uniform" phase. The transition will happen for

$$
a \sim 1 \text { or } k \sim N \sim v
$$

So for $V_{1}=-k / 2$ of order $-v$ a phase transition would occur. Our droplet oscillator analysis sharpens this intuition into an exact statement.

A modified argument works for a general mode $V_{n}$. The potential now has $n$ minima on the circle. Close to each minimum the potential is

$$
V\left(x+\frac{2 \pi}{n}\right)=2 V_{n} \cos (n x) \simeq 2 V_{n}-n^{2} x^{2}+\cdots
$$

For large negative $V_{n}=-k / 2$ the energetically favorable configuration for the ground state is for the particles to distribute equally around $n$ equidistant points on the circle, with $N / n$ particles around each point. Each particle still feels a harmonic oscillator potential of strength $N n^{2} k$, since all other particles contribute to the potential irrespective on which of the $n$ points on the circle they are. The distribution of particles around each of the $n$ points is again a Wigner semicircle, but now with an oscillator strength $N n^{2} k$ and a number of particles $N / n$. The Wigner radius is, then,

$$
a_{n}=\left(\frac{4 N}{n^{4} k}\right)^{\frac{1}{4}}=\frac{1}{n}\left(\frac{4 N}{k}\right)^{\frac{1}{4}}
$$

A phase transition will occur when the semicircles start 'merging', which will happen when their size becomes of order $2 \pi / n$ (as there are $n$ of them). That is, when

$$
a_{n} \sim \frac{1}{n} \quad \text { or } \quad k \sim N
$$

So we obtain the same critical value for $V_{n}$ as for $V_{1}$. In the presence of more than one nonzero $V_{n}$, of course, the argument becomes more complicated. The droplet analysis, however, shows that a phase transition occurs when any of the $V_{n}$ reaches the critical value $V_{n}=-v / 2$.

It should also be clear that the above phase transition is an essentially nonperturbative effect. Indeed, the zero-point fluctuations of $a_{n}$ and $b_{n}$ in the ground state are always of order $\sqrt{n}$, but the fluctuations of $A_{n}$ and $B_{n}$ (and thus also of $R_{n}$ and $\bar{R}_{n}$ ) are of order $\sqrt{n} \operatorname{ch} \theta$. As $V_{n}$ approaches $-v / 2$, these fluctuations diverge and become of order $N$. At that point, droplet fluctuations become large enough to mix the two chiral components and signal the onset of nonperturbative effects. 


\section{Conclusions}

The droplet operator method is an intuitively appealing approach to bosonization and, as demonstrated in the above analysis, can be carried out to produce perturbatively exact results for finite $N$ and probe interesting many-body physics.

An important open question is the applicability of similar methods to higher dimensions. It was suggested in [8] that an adaptation of this method into a 'noncommutative' higher dimensional chiral field would provide a perturbatively exact bosonization in higher dimensions. The irreducible representation space of this algebra was demonstrated to reproduce the Hilbert space of the many-body fermion system, and the states and energies of fermions in a twodimensional hermonic trap were correctly reproduced by the model. What is still missing is an exact mapping of operators that would provide a complete dictionary between the two descriptions. That is, a droplet expression for the density operator, which is a universal operator of the system, is lacking. A possible approach to achieve this would be to work in analogy with nonabelian bosonization and view the extra dimensions as a (particularly large!) 'internal' space of one-dimensional fermions. Some encouraging results in this direction were obtained but the problem is still open and deserves further study.

\section{References}

[1] F. Bloch, Z. Phys. 81, 363 (1933); S. Tomonaga, Prog. Theor. Phys. 5, 544 (1950).

[2] W. Thirring, Ann. Phys. (N.Y.) 3, 91 (1958); J. M. Luttinger, J. Math. Phys. 4, 1154 (1963); D. Mattis and E. Lieb, J. Math. Phys. 6, 304 (1965); S. R. Coleman, Phys. Rev. D 11, 2088 (1975); S. Mandelstam, Phys. Rept. 23 (1976) 307.

[3] E. Witten, Commun. Math. Phys. 92, 455 (1984).

[4] J. Polchinski, Nucl. Phys. B 362, 125 (1991); S. Iso, D. Karabali and B. Sakita, Phys. Lett. B 296, 143 (1992) [arXiv:hep-th/9209003]; B. Sakita, Phys. Lett. B 387, 118 (1996) [arXiv:hep-th/9607047]; D. Karabali and V. P. Nair, Nucl. Phys. B 679, 427 (2004) [arXiv:hep-th/0307281]; 697, 513 (2004) [arXiv:hep-th/0403111].

[5] A. P. Polychronakos, Nucl. Phys. B 705, 457 (2005) [arXiv:hep-th/0408194]; Nucl. Phys. B 711, 505 (2005) [arXiv:hep-th/0411065].

[6] A. Jevicki and B. Sakita, Nucl. Phys. B 165, 511 (1980); D. Karabali and B. Sakita, Int. J. Mod. Phys. A 6, 5079 (1991); S. R. Das, A. Dhar, G. Mandal and S. R. Wadia, Mod. Phys. Lett. A 7, 71 (1992) [arXiv:hep-th/9111021]; Int. J. Mod. Phys. A 8, 325 (1993) [arXiv:hep-th/9204028].

[7] A. Dhar, G. Mandal and N. V. Suryanarayana, JHEP 0601, 118 (2006) [arXiv:hepth/0509164]; A. Dhar and G. Mandal, Phys. Rev. D 74, 105006 (2006) [arXiv:hepth/0603154].

[8] A.P. Polychronakos, Phys. Rev. Lett. 96, 186401 (2006) [arXiv:hep-th/0502150]. 
[9] A. Luther, Phys. Rev. B 19, 320 (1979); F. D. M. Haldane, Varenna 1992 Lectures and cond-mat/0505529; A. Houghton and J. B. Marston, Phys. Rev. B 48, 7790 (1993) [arXiv:cond-mat/9210007]; A. H. Castro Neto and E. Fradkin, Phys. Rev. Lett. 72, 1393 (1994) [arXiv:cond-mat/9304014]; Phys. Rev. B 49, 10877 (1994) [arXiv:cond-mat/9307005]; D. Schmeltzer and A.R. Bishop, Phys. Rev. B 50, 12733 (1994); D. V. Khveshchenko, Phys. Rev. B 52, 4833 (1995) [arXiv:cond-mat/9409118]; D. Schmeltzer, Phys. Rev. 54, 10269 (1996); D. Karabali, Nucl. Phys. B 750, 265 (2006) [arXiv:hep-th/0605006].

[10] A. Enciso and A.P. Polychronakos, Nucl. Phys. B 751, 376 (2006) [arXiv:hep-th/0605040].

[11] D. B. Fairlie and C. K. Zachos, Phys. Lett. B 224, 101 (1989). 